\title{
A New Color Filter Array with Optimal Properties for Noiseless and Noisy Color Image Acquisition
}

\author{
Laurent Condat
}

\begin{abstract}
Digital color cameras acquire color images by means of a sensor on which a color filter array (CFA) is overlaid. The Bayer CFA dominates the consumer market, but there has been recently a renewed interest for the design of CFAs [2]-[6]. However, robustness to noise is often neglected in the design, though it is crucial in practice. In this work, we present a new $2 \times 3$-periodic CFA which provides, by construction, the optimal tradeoff between robustness to aliasing, chrominance noise and luminance noise. Moreover, a simple and efficient linear demosaicking algorithm is described, which fully exploits the spectral properties of the CFA. Practical experiments confirm the superiority of our design, both in noiseless and noisy scenarios.
\end{abstract}

Index Terms-Color filter array (CFA), color imaging, demosaicking, digital camera pipeline, spatio-spectral sampling, luminance/chrominance basis, noise sensitivity.

\section{INTRODUCTION}

The growing popularity of digital photography demands every attempt of improvement in terms of quality and speed of the features provided in digital cameras. The heart of a digital still or video camera is its sensor, a 2-D array of photosites that measure the amount of light absorbed during the exposure time. The color information is obtained by means of a color filter array (CFA) overlaid on the sensor, such that each photosite is covered by a color filter sensitive to only a portion of the visible light spectrum [7]. For a sensor with given geometry, the CFA is the most crucial element in the imaging pipeline that determines the image quality [7], [8]. From the mosaicked image acquired by the camera, some processing is required to recover a full color image with three components per pixel, carrying information in the red (R), green $(\mathrm{G})$ and blue (B) spectral bands to which the human visual system (HVS) is sensitive. This reconstruction operation is called demosaicking, see e.g. [7], [9], [10] and references therein.

The Bayer CFA, which consists in filters with the primary colors $\mathbf{R}, \mathbf{G}, \mathbf{B}$, as depicted in Fig. 1 (a), is the most popular and dominates the consumer market. There is a vast literature dealing with the best way to reduce aliasing artifacts during the demosaicking process, but these artifacts are inherent to the spectral characteristics of the Bayer CFA [11]. Kodak patented new CFAs containing transparent (panchromatic) filters, in addition to R, G, B filters [3], [12]. Other CFAs have been proposed in the literature [4], [5], [13], [14]; some of these

Laurent Condat is with the GREYC in Caen, France (e-mail: laurent.condat@greyc.ensicaen.fr). This work was performed in part during his stay at the Helmholtz Zentrum München - German Research Center for Environmental Health, Neuherberg, Germany, where he was supported by the Marie Curie Excellence Team Grant MEXT-CT-2004-013477, Acronym MAMEBIA, funded by the European Commission.

Part of this work has been presented at the IEEE ICIP Conference [1] are compared in [15]. However, all these CFAs have been designed empirically and are not based on a thorough theory for CFA design. A breakthrough in the field was made by Hirakawa et al., who proposed to design CFAs directly in the Fourier domain, without constraints on the colors of the filters in the spatial domain [2]. Based on previous work characterizing the spectral properties of the Bayer CFA [11] and showing that the mosaicked image actually consists in the superposition of modulated signals encoding the color information, they proposed to design a CFA so that these signals tile the frequency plane with minimum overlap. This paradigm is quite general, however it leaves open questions about the choice of the many parameters of the model. Further insights are provided in [6], [16], [17], where some degrees of freedom in this spatio-spectral formulation of CFA design are re-expressed as solutions of a constrained optimization problem, so as to minimize the norm of the demosaicking operator.

So far, emphasis in CFA design and demosaicking has been put on the minimization of the aliasing artifacts due to spectral overlap of the modulated color channels in the mosaicked image. But with the always increasing resolution of the sensors, aliasing has become a minor issue. In most cases, the optical system is the limiting factor, so that the scene which is sampled by the sensor is bandlimited and moiré artifacts never appear. On the other side, in high-end digital single-lens reflex cameras equipped with expensive and high-quality lenses, an anti-aliasing filter is overlaid on the sensor to get rid of aliasing issues, typically a layer of birefringent material [10], [18]. Thus, in practice, there is no aliasing and even the simplest demosaicking method will do the job of reconstructing the color image; this is on par with the demand of manufacturers for very limited computational burden. Still, robustness to aliasing is an important criterion in CFA design, not because of potential moiré artifacts, but because it determines the intrinsic resolution of the imaging system.

Thus, we argue that robustness to noise is more important than robustness to aliasing. High sensitivity properties allow, when acquiring a given picture, to reduce the exposure time (for less blur due to camera shake), to increase the aperture (for increased depth-of-field, hence less out-of-focus blur), or to use a lower ISO setting and a less destructive denoising process. This is particularly important for photography in low light level environments. Hence, there is a real need for new CFAs with improved sensitivity, so that maximum energy of the color scene is packed into the mosaicked image. However, the sensitivity to noise is investigated in none of the previously cited works on CFA design, although it determines the quality 
of the whole imaging pipeline. In this work, we design a CFA with optimal robustness to aliasing and noise.

The paper is organized as follows. In Section II, we characterize the spatio-spectral properties of CFAs in an appropriate luminance/chrominance basis. In Section III, we express our design requirements and optimize the parameters to yield a new CFA with optimal sensitivity properties and robustness to aliasing. In Section IV, we present a simple and efficient linear demosaicking method, which fully exploits the specificities of the proposed CFA. We show how to extend the approach to optimally handle the presence of noise. In Section V, we validate the superiority of the design by practical experiments in noiseless and noisy situations.

\section{Spectral Characterization of CFAs}

In this article, boldface quantities denote vectors, e.g. $\mathrm{k}=$ $\left[k_{1}, k_{2}\right]^{\mathrm{T}} \in \mathbb{Z}^{2}$ and $\mathbf{0}=[0,0]^{\mathrm{T}}$.

We define a CFA as a color image $\mathbf{c f a}=(\mathbf{c f a}[\mathbf{k}])_{\mathbf{k} \in \mathbb{Z}^{2}}$, where $\mathbf{c f a}[\mathbf{k}]=\left[\operatorname{cfa}^{R}[\mathbf{k}], \operatorname{cfa}^{G}[\mathbf{k}], \operatorname{cfa}^{B}[\mathbf{k}]\right]^{\mathrm{T}} \in[0,1]^{3}$ is the color value in the canonical $\mathbf{R}, \mathbf{G}, \mathbf{B}$ basis, of the filter centered at location $\mathbf{k}$. For instance, a green filter has color $\mathbf{G}=[0,1,0]^{\mathrm{T}}$. The components of $\mathbf{c f a}[\mathbf{k}]$ are constrained to lie in $[0,1]$ for physical realizability, since they correspond to opacity rates: the white color $[1,1,1]^{\mathrm{T}}$ stands for a transparent filter. The CFA is periodic with generating pattern of size $N_{1} \times N_{2}$ if $\mathbf{c f a}\left[k_{1}+N_{1}, k_{2}\right]=\mathbf{c f a}\left[k_{1}, k_{2}+N_{2}\right]=\mathbf{c f a}[\mathbf{k}]$ for every $\mathbf{k} \in \mathbb{Z}^{2}$. We focus in this work on periodic CFAs; thus, the design of CFAs having a random pattern, like in [5], is beyond the scope of this paper. Also, we consider patterns defined on the square lattice, but the principles developed in this work could be extended to other geometries, e.g. an hexagonal arrangement.

We define the color image $\mathbf{i m}=(\mathbf{i m}[\mathbf{k}])_{\mathbf{k} \in \mathbb{Z}^{2}}$ as the ground truth to be estimated by the demosaicking process. That is, $\operatorname{im}[\mathbf{k}]$ is the vector of the three $R, G, B$ values that would have been obtained by the photosite at the location $\mathbf{k}$, if three measurements had been performed using $\mathbf{R}, \mathbf{G}, \mathbf{B}$ filters in front of the sensor. Consequently, the mosaicked image $v=$ $(v[\mathbf{k}])_{\mathbf{k} \in \mathbb{Z}^{2}}$ is such that

$$
v[\mathbf{k}]=\operatorname{im}[\mathbf{k}]^{\mathrm{T}} \mathbf{c f a}[\mathbf{k}] \quad \forall \mathbf{k} \in \mathbb{Z}^{2} .
$$

Note that this model holds in the ideal noise free situation. In practice, an additive random term modeling the effect of noise has to be added in (1) [19]. We come back to the noisy case in Sect. IV-C.

It is well known that in natural images, the R, G, B components are not independent [7], [20]-[23]. Thus, we define the orthonormal basis corresponding to luminance, green/magenta and red/blue chrominance, as

$\mathbf{L}=\frac{1}{\sqrt{3}}[1,1,1]^{\mathrm{T}}, \mathbf{C}_{1}=\frac{1}{\sqrt{6}}[-1,2,-1], \mathbf{C}_{2}=\frac{1}{\sqrt{2}}[1,0,-1]^{\mathrm{T}}$,

We denote $u^{L}, u^{C_{1}}$, and $u^{C_{2}}$ the components of a color image $\mathbf{u}$ in this basis. The proposed basis is arbitrary but convenient to describe the spectral properties of the Bayer CFA [11]. Also, the components of natural images can be considered as statistically independent in this basis, in first approximation [20]. It is important to work with an orthonormal basis, so that the image components are maximally decorrelated. Anyway, the basis is used to formulate the optimization problem, but the proposed CFA is independent on the particular choice of chrominance axes. Hirakawa et al. [2] work with the $\mathbf{G}, \mathbf{R}-\mathbf{G}, \mathbf{B}-\mathbf{G}$ basis, which does not decorrelate the image channels at best; also, the chrominance channels in this basis don't have minimum bandwidth, so that the analysis of aliasing is biased.

Alleysson et al. showed that the mosaicked image $v$ can be interpreted, in the Fourier domain, as the sum of the luminance and chrominance components of the color reference image im, moved at different locations of the frequency plane [11]. We can extend this characterization to every CFA, by writing cfa as the sum of its Fourier components:

$$
\begin{array}{r}
\operatorname{cfa}^{X}[\mathbf{k}]=\sum_{n_{1}=\left\lfloor\frac{N_{1}-1}{2}\right\rfloor}^{\left\lfloor\frac{N_{1}}{2}\right\rfloor} \sum_{n_{2}=0}^{\left\lfloor\frac{N_{2}}{2}\right\rfloor} \alpha_{\mathbf{n}}^{X} \cos \left(\frac{2 \pi n_{1}}{N_{1}} k_{1}+\frac{2 \pi n_{2}}{N_{2}} k_{2}\right)+ \\
\beta_{\mathbf{n}}^{X} \sin \left(\frac{2 \pi n_{1}}{N_{1}} k_{1}+\frac{2 \pi n_{2}}{N_{2}} k_{2}\right)
\end{array}
$$

for every $X \in\left\{L, C_{1}, C_{2}\right\}$ and $\mathbf{k} \in \mathbb{Z}^{2}$, where $\lfloor\cdot\rfloor$ is the rounding operator to the nearest smaller integer. So, designing cfa amounts to choosing its $3 N_{1} N_{2}$ Fourier coefficients $\alpha_{\mathbf{n}}^{X}$ and $\beta_{\mathbf{n}}^{X}$ appropriately. For this, we express the Fourier transform $\hat{v}(\boldsymbol{\omega})=\sum_{\mathbf{k} \in \mathbb{Z}^{2}} v[\mathbf{k}] e^{-j \boldsymbol{\omega}^{\mathrm{T}} \mathbf{k}}$ in function of the Fourier transforms of the components of im:

$$
\begin{aligned}
& \hat{v}(\boldsymbol{\omega})=\sum_{X \in\left\{L, C_{1}, C_{2}\right\}} \sum_{n_{1}=\left\lfloor\frac{N_{1}-1}{2}\right\rfloor} \sum_{n_{2}=0}^{\left\lfloor\frac{N_{1}}{2}\right\rfloor} \\
& \frac{\alpha_{\mathbf{n}}^{X}}{2}\left(\widehat{\mathrm{im}^{X}}\left(\boldsymbol{\omega}+\left[\frac{2 \pi n_{1}}{N_{1}}, \frac{2 \pi n_{2}}{N_{2}}\right]^{\mathrm{T}}\right)+\widehat{\mathrm{im}^{X}}\left(\boldsymbol{\omega}-\left[\frac{2 \pi n_{1}}{N_{1}}, \frac{2 \pi n_{2}}{N_{2}}\right]^{\mathrm{T}}\right)\right)+ \\
& \frac{\beta_{\mathbf{n}}^{X}}{2 j}\left(\widehat{\mathrm{im}^{X}}\left(\boldsymbol{\omega}+\left[\frac{2 \pi n_{1}}{N_{1}}, \frac{2 \pi n_{2}}{N_{2}}\right]^{\mathrm{T}}\right)-\widehat{\mathrm{im}^{X}}\left(\boldsymbol{\omega}-\left[\frac{2 \pi n_{1}}{N_{1}}, \frac{2 \pi n_{2}}{N_{2}}\right]^{\mathrm{T}}\right)\right)
\end{aligned}
$$

for every $\omega \in \mathbb{R}^{2}$. So, whatever the CFA, the Fourier transform of the mosaicked image is the sum of the luminance and chrominance components $\widehat{\mathrm{im}^{X}}$, replicated at the sites of the dual lattice induced by the periodicity of the pattern.

In the spatial domain, this corresponds to writing $v$ as the sum of the luminance and chrominance channels of im, each one being modulated by some specific carrier wave: expanding (1) in the $\mathbf{L}, \mathbf{C}_{1}, \mathbf{C}_{2}$ basis, we get

$$
\begin{array}{r}
v[\mathbf{k}]=\sum_{X \in\left\{L, C_{1}, C_{2}\right\}} \operatorname{im}^{X}[\mathbf{k}] \times \\
\sum_{n_{1}=\left\lfloor\frac{N_{1}-1}{2}\right\rfloor} \sum_{n_{2}=0}^{\left\lfloor\frac{N_{2}}{2}\right\rfloor} \alpha_{\mathbf{n}}^{X} \cos \left(\frac{2 \pi n_{1}}{N_{1}} k_{1}+\frac{2 \pi n_{2}}{N_{2}} k_{2}\right)+ \\
\beta_{\mathbf{n}}^{X} \sin \left(\frac{2 \pi n_{1}}{N_{1}} k_{1}+\frac{2 \pi n_{2}}{N_{2}} k_{2}\right) .
\end{array}
$$

The major contribution of Hirakawa et al. to the problem of CFA design was the idea of directly designing the CFA in the 
Fourier domain by optimizing the carrier waves, so that the baseband luminance is at the origin and the chrominance is modulated far away from it [2]. This constrains the degrees of freedom $N_{1}, N_{2}, \alpha_{\mathbf{n}}^{X}, \beta_{\mathbf{n}}^{X}$ to some extent, but the question of further defining the many remaining parameters is left open. The aim of this work is to tune the parameters to obtain a CFA with optimal robustness to sensor noise.

\section{CFA DESIGN AS A CONSTRAined OptimizATION PROBLEM AND ITS SOLUTION}

\section{A. A Class of CFAs Robust to Aliasing}

We construct, step by step, the structure of a CFA with desirable properties, enforced as design criteria in the frequency domain. Thus, we obtain a class of CFAs parameterized by a small set of variables. We discuss the optimization of these remaining parameters in Sect. III-B.

The requirements we adopt are the following:

Condition on the luminance. For the CFA to be physically realizable, the values $\mathrm{cfa}^{X}[\mathbf{k}]$ have to lie in $[0,1]$. This implies that the luminance channel $\mathrm{im}^{L}$ appears in the baseband of $v$. Further on, we impose that there is no replica of the luminance at other frequencies than zero, in the spectrum of $v$; in other words, the luminance sensitivity of each color filter is the same:

$$
\operatorname{cfa}^{L}[\mathbf{k}]=\alpha_{\mathbf{0}}^{L}
$$

is a constant. Thereafter, we rewrite this value $\gamma_{L}=\alpha_{0}^{L}$ and call it the luminance gain of the CFA. It lies in the interval $[0, \sqrt{3}]$ and characterizes the average light sensitivity of the CFA. This crucial parameter directly controls the noise level in the luminance of the demosaicked image, as explained in Sect. IV-B. We impose this condition of constant luminance sensitivity to make denoising practical. In fact, having a noise variance which changes from pixel to pixel in the demosaicked image, as is the case with the CFAs proposed in [3], [17], makes subsequent denoising a much more difficult task. Also, having a uniform quantum efficiency accross the image plane reduces the issues of under- and over-saturation of the sensor measurements; it is desirable to have a uniform dynamic range and tone curve.

The Bayer CFA satisfies the condition, with $\mathrm{cfa}^{R}[\mathbf{k}]+\mathrm{cfa}^{G}[\mathbf{k}]+\operatorname{cfa}^{B}[\mathbf{k}]=1$; hence, its luminance gain is $\gamma_{L}=1 / \sqrt{3}$.

Conditions on the chrominance. Since the three luminance and chrominance channels of im are assumed mutually independent, we require that their carrier waves in (5) are orthogonal. So, we will be able to separate them optimally during the demosaicking process. This implies that there is no chrominance in the low-frequency part of $v$. Another consequence is that the CFA let pass, in average, the same amount of R, G, and B light through:

$$
\sum_{k_{1}=1}^{N_{1}} \sum_{k_{2}=1}^{N_{2}} \mathrm{cfa}^{R}[\mathbf{k}]=\sum_{k_{1}=1}^{N_{1}} \sum_{k_{2}=1}^{N_{2}} \mathrm{cfa}^{G}[\mathbf{k}]=\sum_{k_{1}=1}^{N_{1}} \sum_{k_{2}=1}^{N_{2}} \operatorname{cfa}^{B}[\mathbf{k}] \text {. }
$$

The Bayer CFA does not satisfy this requirement, with a sensitivity to green two times higher than the one to blue and red.

Thus, the chrominance of im appears in the high-frequency content of $v$ while the luminance is in the baseband. The quality of the reconstruction process essentially depends on the ability to correctly separate these frequency components of $v$. In order to maximally reduce the overlap between the luminance and chrominance channels in the spectrum of $v$, we impose that there is only one replica of each chrominance band, modulated in quadrature at the same frequency $\pm \boldsymbol{\omega}_{0}$. Moreover, we require that the gain of the two chrominance channels is the same, so that the color discrimination of the CFA is the same for every color, without privileged chrominance axis. Consequently, we can rewrite (3) as

$$
\begin{aligned}
& \operatorname{cfa}^{C_{1}}[\mathbf{k}]=\gamma_{C} \sqrt{2} \cos \left(\boldsymbol{\omega}_{0}^{\mathrm{T}} \mathbf{k}-\varphi\right) \\
& \operatorname{cfa}^{C_{2}}[\mathbf{k}]=\gamma_{C} \sqrt{2} \sin \left(\boldsymbol{\omega}_{0}^{\mathrm{T}} \mathbf{k}-\varphi\right),
\end{aligned}
$$

for every $\mathbf{k} \in \mathbb{Z}^{2}$, where we introduce $\gamma_{C}$, the chrominance gain of the CFA, and the phase $\varphi$ is, at this point, a degree of freedom. We will see in Sect. IV-B that the chrominance gain is directly related to the noise level in the chrominance of the demosaicked image. We remark that there is no overlap between the two channels of chrominance, since they occupy the same frequency band, but with phases in quadrature. In comparison with designs where the chrominance is spread at several frequencies, like in [2], the risk of inter-chrominance aliasing is drastically reduced, see the example in Fig. 3. Also, the CFA is independent on the choice of the green-magenta and red-blue chrominance axes, only chosen to express the formulas.

The Bayer CFA does not satisfy these conditions neither, with the chrominance spread at the frequencies $[\pi, 0]^{\mathrm{T}},[0, \pi]^{\mathrm{T}}$, $[\pi, \pi]^{\mathrm{T}}[11]$.

Further on, the modulation frequency $\boldsymbol{\omega}_{0}$ for the chrominance should be the farthest from $\mathbf{0}$, so that the overlap between luminance and chrominance in the spectrum of $v$ is minimal. So, like in [2], we impose that $\boldsymbol{\omega}_{0}$ lies on the boundary of the Nyquist band $[-\pi, \pi]^{2}$. Without restriction, this amounts to have $\boldsymbol{\omega}_{0}=\left[\pi, \omega_{0}\right]^{\mathrm{T}}$-the other choice corresponds to rotating the CFA by $90^{\circ}$. Hence, we have $N_{1}=2$ and the CFA has a $2 \times N_{2}$ periodicity.

We can now rewrite the generic expression of a CFA satisfying the previous conditions as:

$$
\begin{aligned}
\operatorname{cfa}^{L}[\mathbf{k}] & =\gamma_{L} \\
\operatorname{cfa}^{C_{1}}[\mathbf{k}] & =\gamma_{C}(-1)^{k_{1}} \sqrt{2} \cos \left(\omega_{0} k_{2}-\varphi\right) \\
\operatorname{cfa}^{C_{2}}[\mathbf{k}] & =\gamma_{C}(-1)^{k_{1}} \sqrt{2} \sin \left(\omega_{0} k_{2}-\varphi\right),
\end{aligned}
$$

where $\omega_{0}=2 \pi n_{2} / N_{2}$ for some integer $n_{2} \in 1 \ldots\left(N_{2}-1\right) / 2$ relatively prime with $N_{2}$, and $N_{2}, n_{2}, \gamma_{L}, \gamma_{C}, \varphi$ are free parameters. We note that $N_{2} \geq 3$, since there is no $2 \times 2 \mathrm{CFA}$ satisfying the previous criteria.

We remark that $\gamma_{L}$ should be in the range $[\sqrt{3} / 2, \sqrt{3}]$, since there is no reason to prefer a darker CFA over a brighter one, all other things being equal. Indeed, if $\gamma_{L}<\sqrt{3} / 2$, we can replace each color filter value $\mathrm{cfa}^{X}[\mathbf{k}]$ by $1-\mathrm{cfa}^{X}[\mathbf{k}], \forall X \in$ 


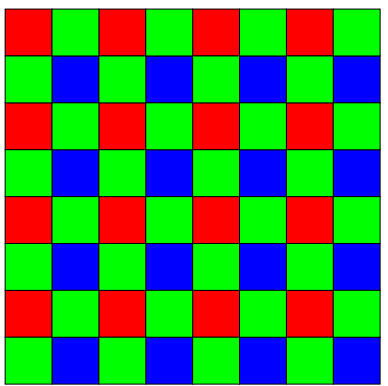

(a) Bayer

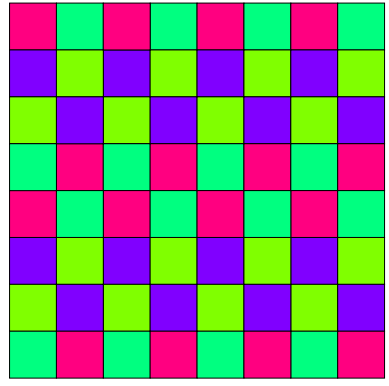

(b) Hirakawa

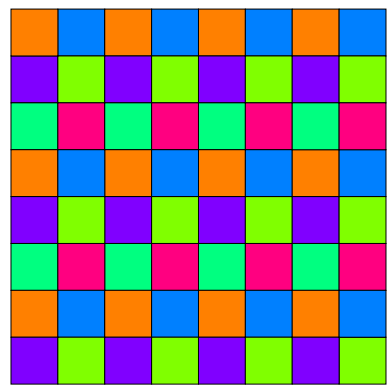

(c) Proposed

Fig. 1. The three CFAs compared in this work: (a) the Bayer pattern, (b) Hirakawa's pattern with 4 colors [2], (c) the proposed $2 \times 3$ pattern with 6 colors.

$\{R, G, B\}, \forall \mathbf{k} \in \mathbb{Z}^{2}$. This does not change the chrominance carrier waves, up to a change of sign, and only the luminance gain $\gamma_{L}$ is turned into $\sqrt{3}-\gamma_{L}$.

\section{B. Optimizing the Left Parameters to Maximize the Sensitivity of the CFA}

We now optimize the parameters in the expressions (10)(12) to maximize the luminance and chrominance gains $\gamma_{C}$ and $\gamma_{L}$. This way, the aliasing artifacts are reduced in the demosaicked image (the chrominance information is less polluted by the high frequency content of the luminance) and the noise level in the demosaicked image is reduced as well. After some calculations given in the Appendix, we obtain the optimal CFA having the desired features, depicted in Fig. 1 (c). It has a small $2 \times 3$ periodicity and a small number (six) of distinct filters, with colors $\left[1,0, \frac{1}{2}\right],\left[1, \frac{1}{2}, 0\right],\left[0,1, \frac{1}{2}\right],\left[0, \frac{1}{2}, 1\right]$, $\left[\frac{1}{2}, 0,1\right]$ and $\left[\frac{1}{2}, 1,0\right]$. Its modulation frequency

$$
\boldsymbol{\omega}_{0}=[\pi, 2 \pi / 3]^{\mathrm{T}}
$$

is well placed: it is far from the origin, to minimize aliasing between the luminance and chrominance information. Also, it is far from the vertical and horizontal axes, where the luminance power spectrum density is higher, due to the predominance of vertical and horizontal structures in natural scenes. The luminance and chrominance gains are $\gamma_{L}=\sqrt{3} / 2$ and $\gamma_{C}=1 / 2$, respectively.

\section{The Demosaicking Strategy and its Properties}

\section{A. Demosaicking by Frequency Selection in the Noiseless Case}

The proposed CFA has a natural and simple demosaicking algorithm associated to it, inspired by its characteristics in the Fourier domain. This demosaicking process amounts to separate the frequency content of the mosaicked image into the luminance and chrominance channels of the reconstructed image. Demosaicking by frequency selection was first explained by Dubois, for the Bayer CFA [24]. Let us recall his method, with our notations. Demosaicking by frequency selection consists in the following steps, where we denote by dem the demosaicked image, which aims at estimating im.

\section{Demosaicking algorithm for the Bayer CFA}

1) Compute the image $v_{1}$ from $v$ by modulation with the carrier wave of the chrominance $C_{1}$, which is modulated at the frequency $[\pi, \pi]^{\mathrm{T}}$, with the gain $\gamma_{C_{1}}=\sqrt{6} / 4$ :

$$
v_{1}[\mathbf{k}]=(-1)^{k_{1}+k_{2}+1} / \gamma_{C_{1}} v[\mathbf{k}] .
$$

2) Apply an appropriate low-pass filter $h_{1}: \operatorname{dem}^{C_{1}}=v_{1} *$ $h_{1}$.

3) The red-blue chrominance $C_{2}$ is present in two independent replicas in the spectrum of $v$. So, first compute the two modulated images $v_{2, H}$ and $v_{2, V}$ to shift in the baseband the chrominance modulated at the frequencies $[\pi, 0]^{\mathrm{T}}$ and $[0, \pi]^{\mathrm{T}}$, respectively:

$$
\begin{aligned}
& v_{2, H}[\mathbf{k}]=2 \sqrt{2}(-1)^{k_{1}} v[\mathbf{k}], \\
& v_{2, V}[\mathbf{k}]=2 \sqrt{2}(-1)^{k_{2}} v[\mathbf{k}] .
\end{aligned}
$$

4) Apply an appropriate low-pass filter $h_{2}$ : $\operatorname{dem}_{H}^{C_{2}}=v_{2, H} *$ $h_{2}$ and $\operatorname{dem}_{V}^{C_{2}}=v_{2, V} *\left(h_{2}\right)^{\mathrm{T}}$, where $\left(h_{2}\right)^{\mathrm{T}}$ is the filter $h_{2}$ rotated by $90^{\circ}$.

5) Average the two estimates of the red-blue chrominance to yield $\operatorname{dem}^{C_{2}}=\left(\operatorname{dem}_{H}^{C_{2}}+\operatorname{dem}_{V}^{C_{2}}\right) / 2$.

6) Estimate the luminance as the residual information content of $v$ by subtracting the re-modulated chrominance:

$$
\begin{array}{r}
\operatorname{dem}^{L}[\mathbf{k}]= \\
=\left(v[\mathbf{k}]+\gamma_{C_{1}}(-1)^{k_{1}+k_{2}} \operatorname{dem}^{C_{1}}[\mathbf{k}]-\right. \\
\left.\frac{\sqrt{2}}{4}\left((-1)^{k_{1}}+(-1)^{k_{2}}\right) \operatorname{dem}^{C_{2}}[\mathbf{k}]\right) / \gamma_{L}
\end{array}
$$

where $\gamma_{L}=1 / \sqrt{3}$.

7) Compute $\operatorname{dem}^{R}, \operatorname{dem}^{G}, \operatorname{dem}^{B}$ by pixelwise change of basis from $\operatorname{dem}^{C_{1}}, \operatorname{dem}^{C_{2}}, \operatorname{dem}^{L}$.

The proposed method for our new CFA is based on the same principles, but the algorithm is even simpler because we don't have to deal with the redundancy of the red-blue chrominance information, a characteristic of the Bayer CFA [11].

\section{Demosaicking algorithm for the proposed CFA}

1) Compute the image $v_{1}$ from $v$ by modulation with the carrier wave of the chrominance $C_{1}$ :

$$
v_{1}[\mathbf{k}]=(-1)^{k_{1}+1} \sqrt{2} / \gamma_{C} \sin \left(2 \pi k_{2} / 3\right) v[\mathbf{k}]
$$

2) Apply an appropriate low-pass filter $h: \operatorname{dem}^{C_{1}}=v_{1} * h$. 
3) Compute the image $v_{2}$ from $v$ by modulation with the carrier wave of the chrominance $C_{2}$ :

$$
v_{2}[\mathbf{k}]=(-1)^{k_{1}} \sqrt{2} / \gamma_{C} \cos \left(2 \pi k_{2} / 3\right) v[\mathbf{k}] .
$$

4) Apply the same low-pass filter $h$ : $\operatorname{dem}^{C_{2}}=v_{2} * h$.

5) Estimate the luminance by subtraction of the remodulated chrominance:

$$
\begin{gathered}
\operatorname{dem}^{L}[\mathbf{k}]=\left(v[\mathbf{k}]+\gamma_{C}(-1)^{k_{1}} \sqrt{2} \sin \left(2 \pi k_{2} / 3\right) \operatorname{dem}^{C_{1}}[\mathbf{k}]\right. \\
\left.-\gamma_{C}(-1)^{k_{1}} \sqrt{2} \cos \left(2 \pi k_{2} / 3\right) \operatorname{dem}^{C_{2}}[\mathbf{k}]\right) / \gamma_{L}
\end{gathered}
$$

6) Compute $\operatorname{dem}^{R}, \operatorname{dem}^{G}, \operatorname{dem}^{B}$ by pixelwise change of basis from $\operatorname{dem}^{C_{1}}, \operatorname{dem}^{C_{2}}, \operatorname{dem}^{L}$.

We remark that if $h$ is sufficiently lowpass, the step 5) is equivalent to a convolution: $\operatorname{dem}^{L}=v * g$, where

$$
g[\mathbf{k}]=\left(\delta_{\mathbf{k}, \mathbf{0}}-(-1)^{k_{1}} \cos \left(2 \pi k_{2} / 3\right) h[\mathbf{k}]\right) / \gamma_{L}
$$

and the Kronecker symbol is defined by $\delta_{x, y}=\{1$ if $x=$ $y, 0$ else $\}$. This can be interesting computationally if the luminance is to be computed in parallel with the chrominance.

We refer to [24] for more details about demosaicking by frequency selection. The proposed algorithm can be easily adapted to handle other CFAs, simply by using the appropriate carrier waves for the chrominance in steps 1), 3), 5). For the $2 \times 4$ CFA proposed by Hirakawa et al. [2], the method reverts to the one detailed in [2], [25]. Note, however, that for the Bayer and Hirakawa CFAs, the estimation of the luminance is not equivalent to a convolution any more. With the proposed CFA, the complexity of the demosaicking process is limited to two convolutions. In addition, they use the same filter and can be performed in parallel. By contrast, with the Bayer CFA, three convolutions with distinct filters must be performed [24].

The choice of the filter $h$ still has to be discussed. In this work, for comparison purpose between the CFAs, we use, for every CFA, the non-separable $13 \times 13$ filter(s) optimal in the least-squares (LS) sense. This methodology has been described for the Bayer CFA in [26]. That is, we minimize the error $\|\mathbf{A x}-\mathbf{b}\|^{2}$, where $\mathbf{x}$ is the vector containing the 169 coefficients, in lexicographic order, of the filter $h$ we are seeking; A is the matrix whose rows contain the 169 pixel values of every $13 \times 13$ patch present in the set of learning images, after the images have been mosaicked and re-modulated (that is, we take the patches of the images $v_{1}$ and $v_{2}$ given by (18) and (19)); $\mathbf{b}$ is the vector containing the true chrominance values for the corresponding center pixel of the patch.The solution of this standard linear algebra problem has the form $\mathbf{x}=\left(\mathbf{A}^{\mathrm{T}} \mathbf{A}\right)^{-1} \mathbf{A}^{\mathrm{T}} \mathbf{b}$. So, the LS-optimal filters are solutions of linear systems of size $169 \times 169$, which have to be solved off-line only once for every CFA. Each linear system can be formed easily by reading the images in scanline, without having to store the matrices $\mathbf{A}$ and $\mathbf{b}$.

In the noiseless case, $9 \times 9$ filters are sufficient, but $13 \times 13$ filters give better results in the noisy case, analyzed in Sect. IVC. In practice, it is much more advantageous to use separable filters. In that case, for our CFA, the vertical filter should have zeros at $\pm 2 \pi / 3$ and the horizontal one at $\pi$. Moreover, IIR recursive filters typically outperform FIR filters, for the same complexity [27].

Hence, by using the same generic method of demosaicking by frequency selection, combined with the LS-optimal lowpass filter(s) for each CFA, we can fairly compare the performances of different CFAs. For the Bayer CFA, non-linear approaches to demosaicking can significantly outperform this linear approach, by exploiting the redundancy of the red-blue chrominance information in the mosaicked image [24]. With our CFA, such an improvement cannot be expected, because there is no such redundancy of the chrominance information.

\section{B. Behavior in the Presence of Noise}

Let us assume that the mosaicked image is corrupted by zero mean additive white Gaussian noise (AWGN):

$$
v[\mathbf{k}]=\mathbf{i m}[\mathbf{k}]^{\mathrm{T}} \mathbf{c f a}[\mathbf{k}]+\sigma \cdot \varepsilon[\mathbf{k}], \quad \forall \mathbf{k} \in \mathbb{Z}^{2},
$$

where $\varepsilon[\mathbf{k}] \sim \mathcal{N}(0,1)$ and $\sigma$ is the noise standard deviation. In practice, the AWGN assumption is not met; real noise is more accurately modeled as the sum of Gaussian and Poissonian noises [19]. Moreover, the pixel values are gamma corrected with respect to the photon counts output by the sensor [28] and this step of tone mapping modifies the noise characteristics. However, homomorphic nonlinear transformations can be efficiently employed for variance stabilization [29], so that the problem can be recast in the AWGN setting.

Let us denote by $\mathcal{D}$ the demosaicking process described in Sect IV-A and by $v_{0}$ the noise free mosaicked image, so that $v=v_{0}+\sigma . \varepsilon$. The linearity of $\mathcal{D}$ is an important property, which allows to exactly characterize the behavior of noise throughout demosaicking. Thus,

$$
\mathcal{D} v=\mathcal{D} v_{0}+\mathcal{D} \varepsilon
$$

and the demosaicked image is corrupted by the additive Gaussian color noise image $\varepsilon=\mathcal{D} \varepsilon$. The demosaicking process consists of modulation with pure sines and convolutions. A pure sine is the sum of two complex exponentials and multiplying by a complex exponential in the spatial domain is equivalent to a shift in the Fourier domain, which leaves the power spectrum density of the noise unchanged. Therefore, we can easily characterize the demosaicked noise $\varepsilon$ : under the assumption that the lowpass filter $h$ is bandlimited with cutoff frequency $\pi / 3$,

- the three channels $\varepsilon^{L}, \varepsilon^{C_{1}}, \varepsilon^{C_{2}}$ of $\varepsilon$ are statistically independant.

- $\varepsilon^{C_{1}}$ and $\varepsilon^{C_{2}}$ have zero mean and power spectrum density $\sigma^{2}|\hat{h}(\boldsymbol{\omega})|^{2} / \gamma_{C}^{2}$.

- $\varepsilon^{L}$ has zero mean and power spectrum density $\sigma^{2}|\hat{g}(\boldsymbol{\omega})|^{2}=\sigma^{2}\left|1-\hat{h}\left(\boldsymbol{\omega}-\boldsymbol{\omega}_{0}\right) / 2-\hat{h}\left(\boldsymbol{\omega}+\boldsymbol{\omega}_{0}\right) / 2\right|^{2} / \gamma_{L}^{2}$.

Thus, we have established that the inverses of $\gamma_{C}$ and $\gamma_{L}$ are the amplification factors of noise in the chrominance and luminance channels of the demosaicked image, respectively. That is why the maximization of the gains is so important and we designed the proposed CFA along this line. 


\section{Joint Demosaicking and Denoising in the Noisy Case}

The development and analysis of a generic strategy for joint demosaicking and denoising is beyond the scope of this paper. However, it is not difficult to modify the generic framework of demosaicking by frequency selection to optimally handle the presence of noise. We developed this strategy for the Bayer CFA and obtained state-of-the-art results in [30]. Here, we apply the same methodology to the proposed CFA.

From the analysis in the last section, we remark that applying the proposed demosaicking process to noisy data yields a noisy demosaicked image, where the noise has been split into the luminance and chrominance channels during reconstruction. We propose to modify the process so that the noise remains in the luminance of the demosaicked image. This is achieved by denoising the chrominance channels before estimating the luminance. For this, we keep the algorithm unchanged but we seek the Wiener-like filter $h$ that estimate the denoised chrominance directly, optimally in the leastsquares sense for a given base of test images. With the same notations as in Sect. IV-A, we now want to minimize the expectation of the error $\mathcal{E}\left\{\|\tilde{\mathbf{A}} \mathbf{x}-\mathbf{b}\|^{2}\right\}$, where $\tilde{\mathbf{A}}$ is equal to $\mathbf{A}$ plus noise, since every re-modulated mosaicked patch is contaminated by noise. We have:

$$
\mathcal{E}\left\{\tilde{\mathbf{A}}^{\mathrm{T}} \mathbf{b}\right\}=\mathbf{A}^{\mathrm{T}} \mathbf{b} \text { and } \mathcal{E}\left\{\tilde{\mathbf{A}}^{\mathrm{T}} \tilde{\mathbf{A}}\right\}=\mathbf{A}^{\mathrm{T}} \mathbf{A}+\sigma^{2} N / \gamma_{C}^{2} \mathbf{I},
$$

where $\mathbf{I}$ is the identity matrix and $N$ is the total number of pixels in the image base (the number of rows of $\mathbf{A}$ ). Therefore, the coefficients of the $13 \times 13$ filter $h$ are given by the vector x solution of the linear system of size $169 \times 169$ :

$$
\left(\frac{1}{N} \mathbf{A}^{\mathrm{T}} \mathbf{A}+\frac{\sigma^{2}}{\gamma_{C}^{2}} \mathbf{I}\right) \mathbf{x}=\frac{1}{N} \mathbf{A}^{\mathrm{T}} \mathbf{b} .
$$

After the denoised chrominance images $\operatorname{dem}^{C_{1}}$ and $\operatorname{dem}^{C_{2}}$ are re-modulated and subtracted from $v$ to estimate the luminance, we have $\operatorname{dem}^{L} \approx \mathrm{im}^{L}+\varepsilon / \gamma_{L}$. Other said, the noise lies almost completely in the luminance of the reconstructed image. More precisely, the luminance noise has zero mean and power spectrum density $\sigma^{2} / \gamma_{L}^{2} \mid 1-\hat{h}\left(\boldsymbol{\omega}-\boldsymbol{\omega}_{0}\right) / 2-\hat{h}(\boldsymbol{\omega}+$ $\left.\boldsymbol{\omega}_{0} / 2\right)\left.\right|^{2}$. Since $h$ is very lowpass, the noise can be considered to be white. Therefore, after demosaicking, we just have to consider $\operatorname{dem}^{L} / \sqrt{3}$ as a grayscale image corrupted by AWGN of variance $\sigma^{2} /\left(\sqrt{3} \gamma_{L}\right)^{2}=4 / 9 \sigma^{2}$ and apply our favorite denoising method to it. By comparison, the noise variance is $\sigma^{2}$ with the Bayer CFA [30].

\section{PERFormance ANALYSis}

In order to evaluate the performances of our new CFA, we compare it to the Bayer CFA and to the $2 \times 4$ CFA proposed by Hirakawa et al. [2], depicted in Fig. 1 (b), which represents the state of the art with respect to noiseless demosaicking simulations.

Let us summarize the spectral properties of these CFAs:

- The spatio-spectral properties of sampling with the Bayer CFA are well established [11]. In our notations, the luminance gain is $\gamma_{L}=1 / \sqrt{3}$. The green/magenta chrominance is modulated at the frequency $[\pi, \pi]^{\mathrm{T}}$ with the chrominance gain $\gamma_{C_{1}}=\sqrt{6} / 4$ and the red/blue

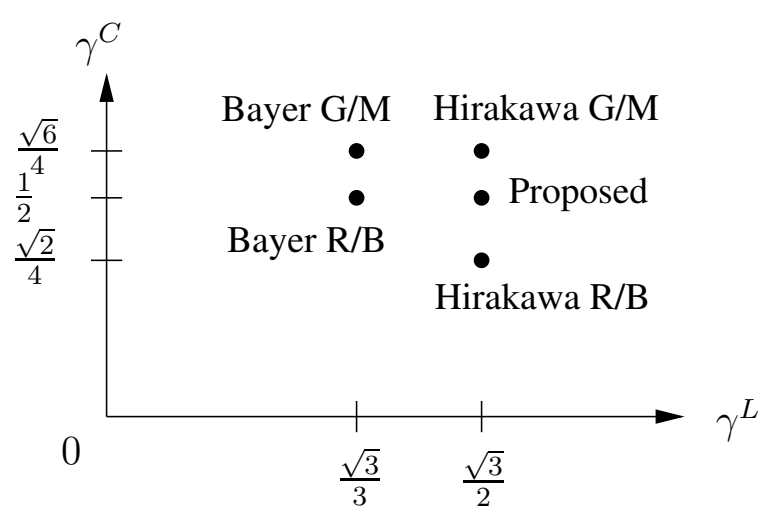

Fig. 2. The Luminance and chrominance gains of the Bayer, Hirakawa and proposed CFAs, depicted in Fig. 1.

chrominance is modulated at $[0, \pi]^{\mathrm{T}}$ and at $[\pi, 0]^{\mathrm{T}}$. The chrominance gain $\gamma_{C_{2}}$ can be defined as the inverse of the noise amplification factor in this channel, when demosaicking using the algorithm given in Sect. IV-A. This yields $\gamma_{C_{2}}=1 / 2$ [30].

- The pattern designed by Hirakawa et al. [2] consists in filters with the colors $\left[\frac{1}{2}, 1,0\right],\left[0,1, \frac{1}{2}\right],\left[1,0, \frac{1}{2}\right]$ and $\left[\frac{1}{2}, 0,1\right]$, arranged with a $2 \times 4$ periodicity. Its luminance gain is $\gamma_{L}=\sqrt{3} / 2$. The green/magenta chrominance is modulated at $[\pi, \pm \pi / 2]^{\mathrm{T}}$ with $\gamma_{C_{1}}=\sqrt{6} / 4$ and the red/blue chrominance is modulated at $[\pi, \pi]^{\mathrm{T}}$ with $\gamma_{C_{2}}=\sqrt{2} / 4$.

- As detailed in Sect. III-B, the proposed $2 \times 3$ CFA has a luminance gain $\gamma_{L}=\sqrt{3} / 2$. The two chrominance bands are modulated at the same frequency $[\pi, \pm 2 \pi / 3]^{\mathrm{T}}$ with the chrominance gain $\gamma_{C}=1 / 2$.

The luminance and chrominance gains of the three compared CFAs are summarized in Fig. 2. The Bayer CFA has high chrominance gains, since it is made of filters with maximally saturated (primary) colors. Hirakawa's and the proposed CFAs have a higher luminance gain; they are globally more sensitive to light. They both capture the same amount of chrominance information: $\left(\gamma_{C_{1}}\right)^{2}+\left(\gamma_{C_{2}}\right)^{2}=1 / 2$. But this is obtained for Hirakawa's CFA at the cost of a strong asymmetry between the two color components: $\gamma_{C_{1}} / \gamma_{C_{2}}=\sqrt{3}$; this means that noise is more amplified in the blue-red band than in the greenmagenta band during demosaicking.

A convenient way to illustrate the modulation of the chrominance in the Fourier domain, induced by mosaicking with a CFA, is to look at the demosaicked images when the initial image im is a gray zoneplate (a radial chirp). The images, given in Fig. 4 for the three considered CFAs, show how the demosaicking method splits the frequency content of the mosaicked image between the luminance and chrominance bands of the reconstructed color image. Due to the particular form of the zoneplate, the instantaneous frequency in the image $\operatorname{im}[\mathbf{k}]$ is proportional to $\mathbf{k}$, so that the aliasing pattern in the demosaicked image illustrates the modulation frequencies in the upper-right quadrant of the Nyquist-band.

We can see in Fig. 4 (c) that Hirakawa's CFA is sensitive to inter-chrominance aliasing between the two chrominance bands. This may be visible in demosaicked images at sharp 


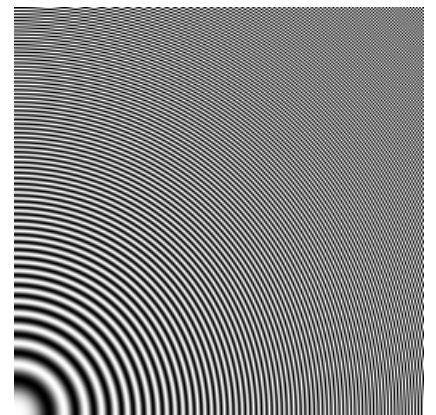

(a) Original

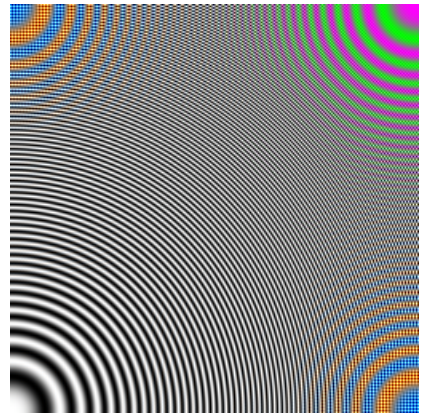

(b) Bayer

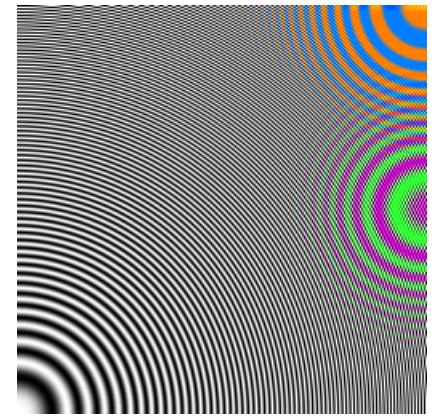

(c) Hirakawa

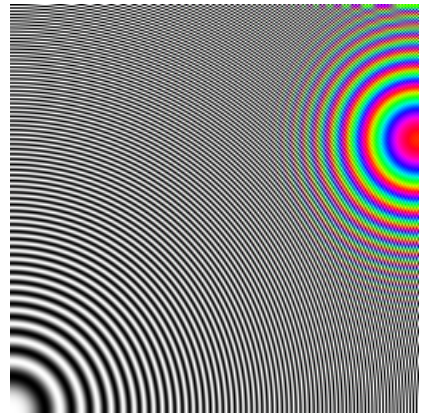

(d) Proposed

Fig. 4. Results of demosaicking by frequency selection with the three CFAs depicted in Fig. 1, on a grayscale synthetic zoneplate pattern.

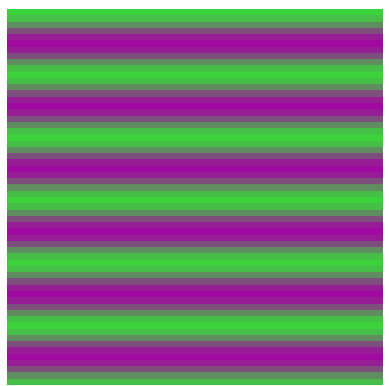

(a) Original

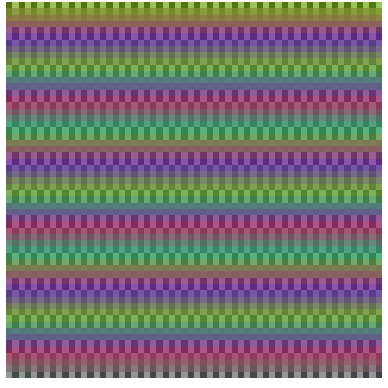

(b) Hirakawa

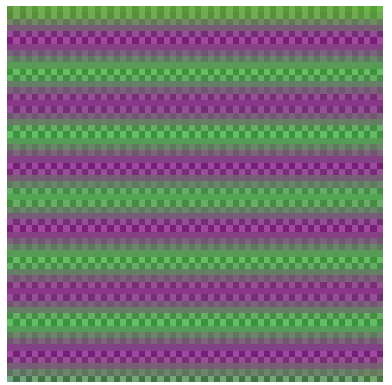

(c) Proposed
Fig. 3. Demosaicking results for the synthetic image (a), which consists in a sine with pulsation $\pi / 5$ oscillating between green and magenta. With the CFA of Hirakawa et al., aliasing between the two chrominance bands appears (b), while with our CFA, there is only aliasing between the chrominance and the luminance (c).

color transitions for horizontally aligned objects. This effect is illustrated by a synthetic example in Fig. 3.

\section{A. Evaluation in Noiseless Situations}

For experimental validation purpose, we consider the classical Kodak test set of 24 color images of size $768 \times 512$. The images were mosaicked using the considered CFAs and demosaicked using frequency selection, as discussed in Sect. IV-A. To simulate an acquisition with a real camera having a fixed sensor, all images were put in landscape mode; that is, the vertical images were turned $90^{\circ}$ left. The $\mathrm{CPSNR}^{1}$ obtained

\footnotetext{
${ }^{1}$ The CPSNR between the color images $\mathbf{u}_{1}$ and $\mathbf{u}_{2}$ is equal to $10 \log _{10}\left(255^{2} / \mathrm{MSE}\right)$ with $\mathrm{MSE}=\sum_{X=R, G, B} \sum_{\mathbf{k}}\left(u_{1}^{X}[\mathbf{k}]-\right.$ $\left.u_{2}^{X}[\mathbf{k}]\right)^{2} /(3 N . M)$ for images of size $N \times M$. A 10 pixel-wide band around the border of the images was ignored when computing the CPSNR, since the initial images used for the tests have been badly acquired at the boundaries. The pixel values were rounded to integers in $0 \ldots 255$ before computing the
} CPSNR.
TABLE I

CPSNR FOR THE DEMOSAICKING EXPERIMENTS USING DIFFERENT

CFAS, IN THE NOISELESS CASE.

\begin{tabular}{|c|c|c|c|c|c|}
\hline \multirow{2}{*}{$\begin{array}{r}\text { Demosa. } \\
\text { method }\end{array}$} & \multicolumn{2}{|c|}{ Non-linear } & \multicolumn{3}{|c|}{$\begin{array}{c}\text { Demosaicking by frequency selection } \\
\text { with } 13 \times 13 \text { LS-optimal filters }\end{array}$} \\
\hline & Bayer & Bayer & Bayer & Hirakawa & Proposed \\
\hline 1 & 38.14 & 37.56 & 37.66 & 40.06 & 39.65 \\
\hline 2 & 40.02 & 40.72 & 39.83 & 40.91 & 40.36 \\
\hline 3 & 41.67 & 42.68 & 41.33 & 41.82 & 42.15 \\
\hline 4 & 40.63 & 40.99 & 40.39 & 40.59 & 40.58 \\
\hline 5 & 37.86 & 38.03 & 37.34 & 37.34 & 36.98 \\
\hline 6 & 40.05 & 38.03 & 38.13 & 41.32 & 40.79 \\
\hline 7 & 42.13 & 42.90 & 41.72 & 41.88 & 42.02 \\
\hline 8 & 35.23 & 35.22 & 33.49 & 37.82 & 37.39 \\
\hline 9 & 42.08 & 42.58 & 41.29 & 42.32 & 42.27 \\
\hline 10 & 42.21 & 42.61 & 41.86 & 42.69 & 42.71 \\
\hline 11 & 39.75 & 39.33 & 38.91 & 40.65 & 40.30 \\
\hline 12 & 43.07 & 42.77 & 42.20 & 43.44 & 43.57 \\
\hline 13 & 35.08 & 33.76 & 34.80 & 35.61 & 35.25 \\
\hline 14 & 35.87 & 37.15 & 35.42 & 36.35 & 36.02 \\
\hline 15 & 39.68 & 39.83 & 39.57 & 39.80 & 39.63 \\
\hline 16 & 43.68 & 41.14 & 40.90 & 44.80 & 44.68 \\
\hline 17 & 41.59 & 41.26 & 41.42 & 41.74 & 41.23 \\
\hline 18 & 37.42 & 37.12 & 37.42 & 37.75 & 37.48 \\
\hline 19 & 40.35 & 40.04 & 38.71 & 41.60 & 41.20 \\
\hline 20 & 40.39 & 41.11 & 40.48 & 41.60 & 41.15 \\
\hline 21 & 38.75 & 38.67 & 38.48 & 40.65 & 40.23 \\
\hline 22 & 38.11 & 38.50 & 37.97 & 38.85 & 38.72 \\
\hline 23 & 42.14 & 43.14 & 41.82 & 42.45 & 41.98 \\
\hline 24 & 35.38 & 34.84 & 35.29 & 36.61 & 36.21 \\
\hline Mean & 39.64 & 39.58 & 39.02 & 40.36 & 40.11 \\
\hline
\end{tabular}

are reported in Tab. I. We also provide the results obtained for the Bayer CFA with two non-linear demosaicking methods among the best in the literature ${ }^{2}$, to illustrate the achievable improvement over linear demosaicking.

The best numerical results are obtained with Hirakawa's CFA, which slightly outperforms the proposed CFA in average. The Bayer CFA is far behind, even when combined with sophisticated non-linear demosaicking. In the illustrations of Fig. 5, we can see that the typical color fringes (see the fence) and zipper effects (see the red bow) that occur with the Bayer CFAs disappear with the two modern CFAs. With the proposed CFA, the aliasing artifacts take the form of rainbow

${ }^{2}$ For the method of Dubois [24], we computed the MSE using the images available online at

http://www.site.uottawa.ca/ edubois/demosaicking/.

For the method of Nai-Xiang et al. [31], we performed the demosaicking experiments using the Matlab code put available online by the authors at http: / / www.ntu.edu.sg/home5/CHAN0069/AFdemosaick. zip 

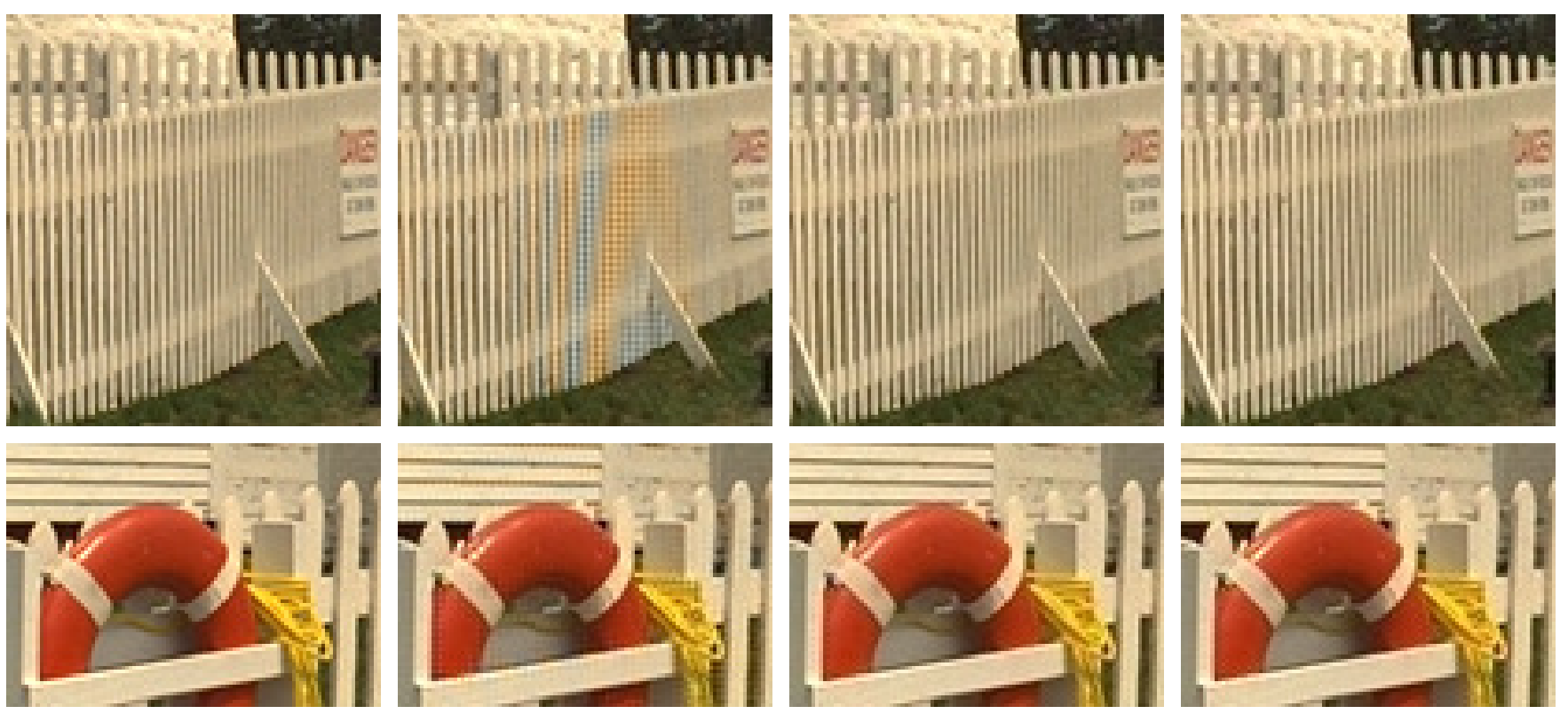

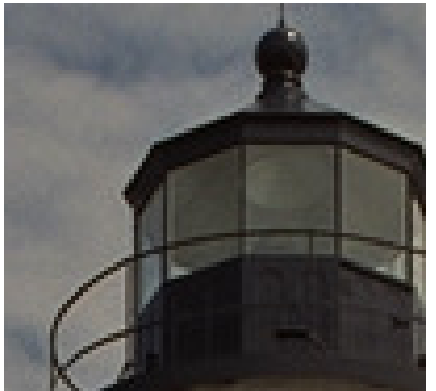

Original

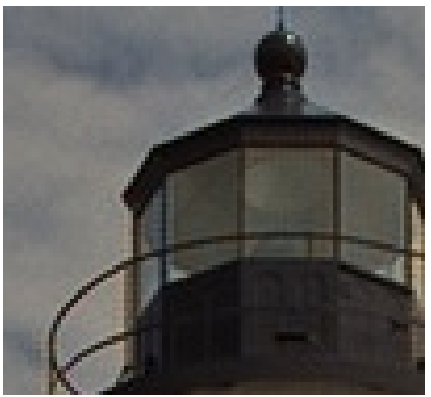

Bayer

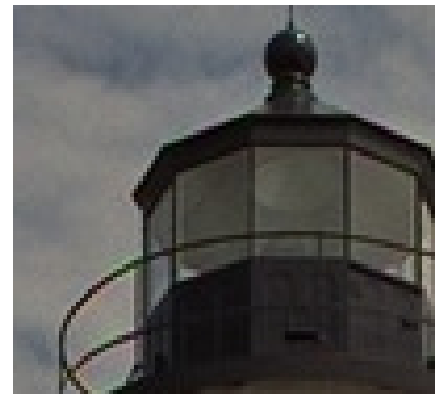

Hirakawa

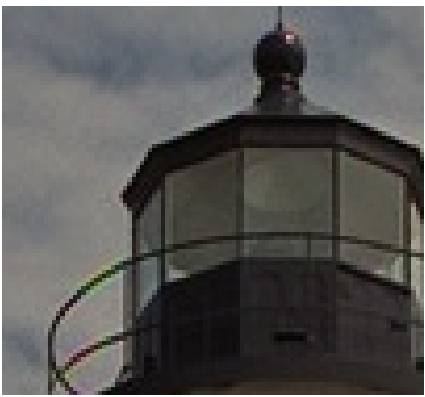

Proposed

Fig. 5. Results of demosaicking by frequency selection used with the three CFAs depicted in Fig. 1, on three parts of the Lighthouse image.

halos with low intensity around sharp transitions oriented at $\pm \arctan (3 / 2) \approx \pm 56^{\circ}$ with respect to the horizontal axis. With Hirakawa's CFA, the artifacts are present around transitions with more orientations than with our CFA, but with lower intensity, and they take the form of bicolored instead of rainbow-like halos.

\section{B. Evaluation in Noisy Situations}

The ideal noiseless scenario is not representative of realistic acquisition conditions. The sensor delivers data corrupted by noise, whose level depends on the analog amplification gain applied before A/D conversion and corresponding to the ISO setting in the camera [19].

First, we illustrate in Fig. 6 the theoretical analysis of Sect. IV-B: the demosaicking process splits the noise into the luminance and chrominance channels of the reconstructed image, with amplification equal to the inverse of the luminance and chrominance gains of the CFA. With Hirakawa's CFA, there is more noise in the blue-red than in the green-magenta chrominance channel, while the proposed CFA is free of this asymmetry in the treatment of the color information. Moreover, the total chrominance noise energy is amplified by a factor $1 / \gamma_{C_{1}}^{2}+1 / \gamma_{C_{2}}^{2}$ and this value is $33 \%$ higher with Hirakawa's CFA than with the proposed CFA. The level of luminance noise is higher with the Bayer CFA than with the two other ones. These properties are true irrespective of the noise variance $\sigma^{2}$.

In Tab. II, we report the joint demosaicking and denoising results obtained with the framework described in Sect. IV-C. The images were mosaicked using the considered CFAs and corrupted with two different noise levels ${ }^{3}$. The state-of-the-art BM3D method [32] was used to denoise the luminance of the demosaicked image. As a result, the proposed CFA slightly outperforms the CFA of Hirakawa et al. . We found out that this superiority holds for a noise level as low as $\sigma=4$, in which case the mean CPSNR over the 24 images is 37.60 vs. 37.58. Moreover, the improvement over the Bayer CFA is very significant. We caution the reader that the comparison of the CFAs may depend on the choice of the reconstruction process, but the superiority of our CFA should be guaranteed by its lowest noise amplification. In Fig. 7, we illustrate the visual quality after demosaicking and denoising; we chose a high noise level $\sigma=40$ to emphasize the differences in this example. The result with the Bayer CFA is corrupted by many denoising artifacts. The details are much better preserved with Hirakawa's and the proposed CFAs. With Hirakawa's CFA, the man's face is too bluish.

\footnotetext{
${ }^{3}$ We used the same noise realization for every method and every image, using the Matlab command randn('state',0); before calling noise=sigma $*$ randn (height, width) ;
} 


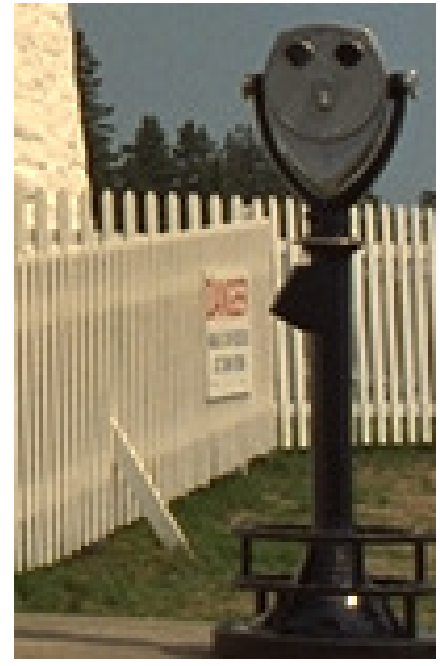

Original

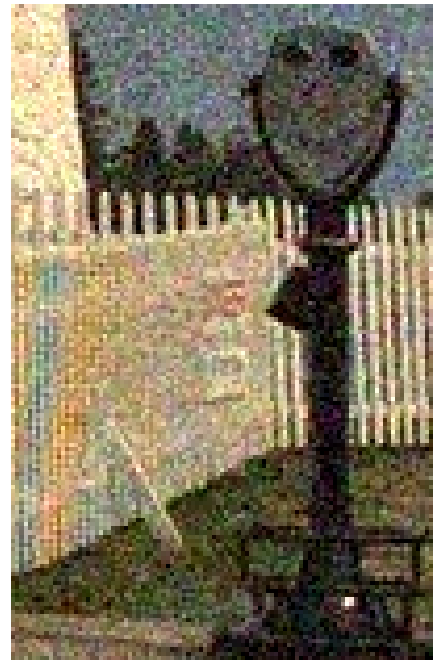

Bayer

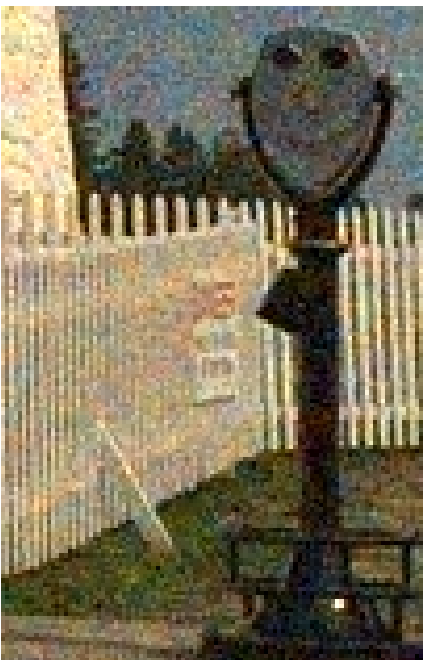

Hirakawa

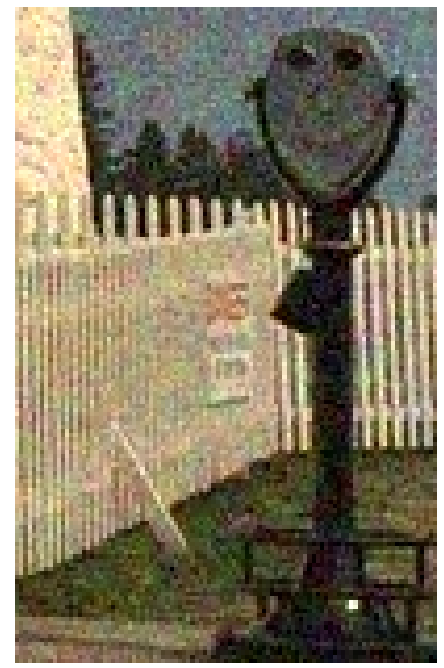

Proposed

Fig. 6. Results of demosaicking by frequency selection, as described in Sect. IV-A, with the three CFAs depicted in Fig. 1, on a part of the Lighthouse image. The mosaicked image were corrupted by additive white Gaussian noise of standard deviation $\sigma=20$ and no denoising process is applied.

TABLE II

CPSNR FOR THE JOINT DEMOSAICKING/DENOISING EXPERIMENTS USING DIFFERENT CFAS, IN THE NOISY CASE.

\begin{tabular}{c|cc|cc|cc} 
CFA & \multicolumn{2}{|c|}{ Bayer } & \multicolumn{2}{c|}{ Hirakawa } & \multicolumn{2}{c}{ Proposed } \\
\hline$\sigma$ & 10 & 20 & 10 & 20 & 10 & 20 \\
\hline 1 & 30.56 & 27.15 & 32.71 & 28.83 & 32.79 & 28.95 \\
2 & 33.05 & 30.63 & 34.12 & 31.30 & 34.30 & 31.54 \\
3 & 34.72 & 31.75 & 35.58 & 32.48 & 35.89 & 32.82 \\
4 & 33.19 & 30.51 & 34.18 & 31.26 & 34.42 & 31.53 \\
5 & 30.83 & 27.43 & 32.09 & 28.81 & 32.29 & 29.00 \\
6 & 31.84 & 28.57 & 33.79 & 30.15 & 33.85 & 30.28 \\
7 & 34.61 & 31.28 & 35.45 & 32.21 & 35.75 & 32.51 \\
8 & 30.19 & 27.51 & 32.52 & 29.25 & 32.61 & 29.38 \\
9 & 34.50 & 31.52 & 35.44 & 32.37 & 35.69 & 32.68 \\
10 & 34.67 & 31.59 & 35.63 & 32.54 & 35.88 & 32.83 \\
11 & 32.25 & 29.20 & 33.81 & 30.47 & 33.94 & 30.65 \\
12 & 34.26 & 31.55 & 35.41 & 32.37 & 35.64 & 32.65 \\
13 & 29.28 & 25.63 & 31.37 & 27.51 & 31.33 & 27.58 \\
14 & 29.80 & 27.08 & 31.00 & 28.11 & 31.12 & 28.25 \\
15 & 33.50 & 30.87 & 34.45 & 31.63 & 34.65 & 31.90 \\
16 & 33.37 & 30.31 & 35.09 & 31.58 & 35.26 & 31.79 \\
17 & 33.96 & 30.90 & 35.15 & 32.00 & 35.28 & 32.22 \\
18 & 31.33 & 28.05 & 32.72 & 29.45 & 32.79 & 29.59 \\
19 & 32.57 & 29.90 & 34.32 & 31.02 & 34.44 & 31.21 \\
20 & 34.29 & 31.50 & 35.45 & 32.34 & 35.61 & 32.57 \\
21 & 32.29 & 28.98 & 34.00 & 30.47 & 34.11 & 30.63 \\
22 & 31.81 & 28.96 & 33.24 & 30.11 & 33.39 & 30.29 \\
23 & 35.14 & 32.21 & 35.65 & 32.65 & 35.93 & 33.01 \\
24 & 31.06 & 28.09 & 32.64 & 29.57 & 32.67 & 29.71 \\
\hline Mean & 32.63 & 29.63 & 33.99 & 30.77 & $\mathbf{3 4 . 1 5}$ & $\mathbf{3 0 . 9 8}$
\end{tabular}

\section{CONCLusion}

In this work, we redefined the problem of CFA design as the maximization of the energy of the color scene encoded in the mosaicked image, through the choice of the gains of the CFA in an orthonormal luminance and chrominance basis. In fact, these gains are the inverse of the noise amplification factors in the luminance and chrominance channels of the demosaicked image. We derived the analytical solution to the optimization of these gains, under the constraint that the chrominance is modulated far away from the luminance in the Fourier domain, for robustness to aliasing. The proposed CFA has six colors and a periodic pattern of size $2 \times 3$. A sensor equipped with the proposed CFA instead of the standard Bayer CFA should provide images with higher perceived resolution (because the anti-alias filter can be removed from the sensor) and better quality (the lower level of noise allows the use of a less destructive denoising method).

In comparison with the recent design of Hirakawa et al. [2], the chrominance noise is less amplified, which yielded slightly better results in our experiments. Also, our CFA is less sensitive to inter-chrominance aliasing and does not favor any chrominance axis.

Moreover, the superiority of our CFA is obtained with the simple, linear and computationally cheap approach to demosaicking by frequency selection. We extended our methodology of joint demosaicking and denoising [30] to the proposed CFA. So, the combination of the new CFA and this new reconstruction framework pushes the state of the art of reconstruction from noisy sensor data to a higher level, at the low cost of two convolutions and denoising a grayscale image, for which real-time algorithms exist [33], [34].

All the numerical and visual results of this paper can be reproduced using the Matlab code available on the homepage of the author. In future work, we will investigate the choice of the precise spectral sensitivity functions of the six colors defining the new CFA, a difficult problem [35].

\section{APPENDIX I}

MAXIMIZATION OF THE LUMINANCE AND CHROMINANCE GAINS

Let us write $\mathrm{cfa}^{i}, i=0,1,2$, instead of $\mathrm{cfa}^{X}, X=R, G, B$, respectively. By expanding the equality $\mathbf{c f a}=\operatorname{cfa}^{L} \mathbf{L}+$ $\operatorname{cfa}^{C_{1}} \mathbf{C}_{1}+\mathrm{cfa}^{C_{2}} \mathbf{C}_{2}$, we obtain:

$\operatorname{cfa}^{i}[\mathbf{k}]=\frac{\gamma_{L}}{\sqrt{3}}+\frac{2 \gamma_{C}}{\sqrt{3}}(-1)^{k_{1}} \sin \left(\frac{2 \pi}{3}(1-i)+\omega_{0} k_{2}-\varphi\right)$. 


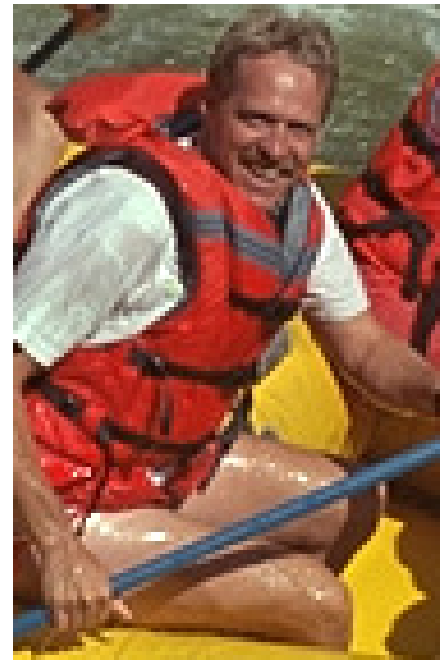

Original

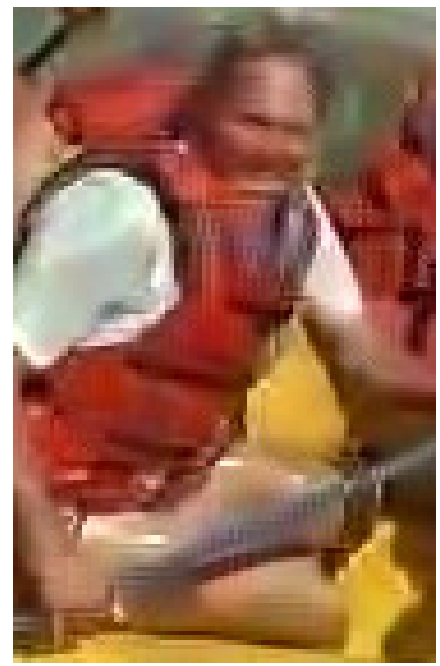

Bayer

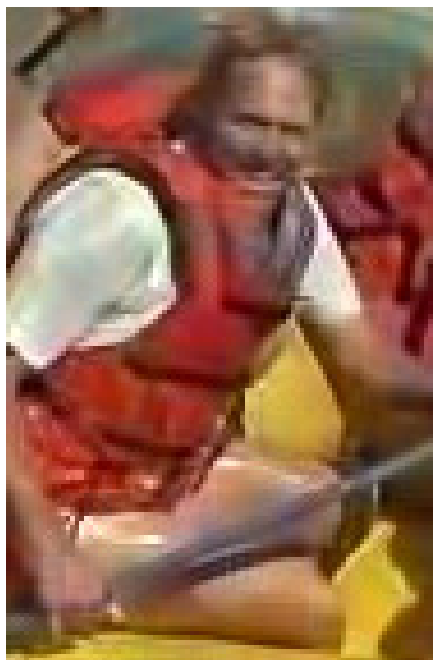

Hirakawa

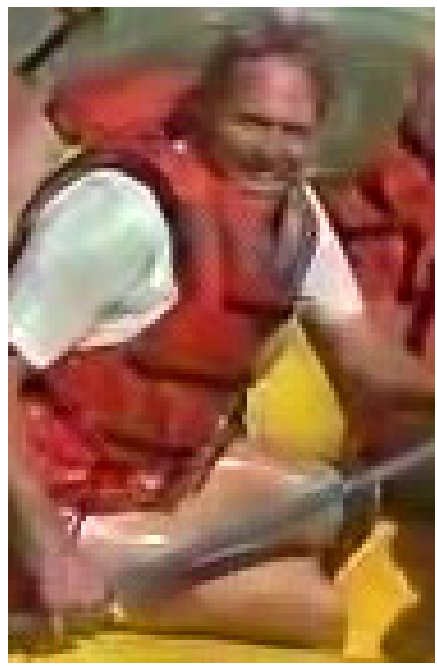

Proposed

Fig. 7. Results of joint demosaicking and denoising, as described in Sect. IV-C, with the three CFAs depicted in Fig. 1, on a part of the Kodak image no. 14. The mosaicked image were corrupted by additive white Gaussian noise of standard deviation $\sigma=40$ and the BM3D denoising method was used.

Let us define the set

$$
\begin{aligned}
\Omega & =\left\{\left|\sin \left(\frac{2 \pi}{3}(1-i)+\omega_{0} k_{2}-\varphi\right)\right| ; i, k_{2} \in \mathbb{Z}\right\} \\
& =\left\{\left|\sin \left(\omega_{1} k-\varphi\right)\right| ; k \in \mathbb{Z}\right\},
\end{aligned}
$$

where

$$
\omega_{1}=\operatorname{gcd}\left(\omega_{0}, \frac{2 \pi}{3}\right)=\frac{2 \pi}{\operatorname{lcm}\left(3, N_{2}\right)},
$$

and gcd and $1 \mathrm{~cm}$ denote the greatest common divisor and the least common multiple, respectively. The identity between (27) and (28) is exactly Bézout's identity.

Since $\gamma_{L}>\sqrt{3} / 2$, the limiting factor for maximizing $\gamma_{C}$ is the set of constraints $\mathrm{cfa}^{i}[\mathbf{k}] \leq 1$ for every $i, \mathbf{k}$. Then, maximizing $\gamma_{C}$ under these constraints amounts to choose $\gamma_{C}$ such that

$$
\frac{\gamma_{L}}{\sqrt{3}}+\frac{2 \gamma_{C}}{\sqrt{3}} \max (\Omega)=1 \Leftrightarrow \gamma_{C}=\frac{\sqrt{3}-\gamma_{L}}{2 \max (\Omega)} .
$$

So, we have to choose $\varphi$ so that $\max (\Omega)$ is minimal. This is the case if and only if

$$
\varphi \in\left\{ \pm \varphi_{0}+m \omega_{1} ; m \in \mathbb{Z}\right\},
$$

where

$$
\varphi_{0}= \begin{cases}\omega_{1} / 4-\pi / 2 & \text { if } N_{2} \text { is odd } \\ \omega_{1} / 2-\pi / 2 & \text { if } N_{2} \text { is even }\end{cases}
$$

For such a $\varphi$, the value $\max (\Omega)$ is

$$
\max (\Omega)=\sin \left(-\varphi_{0}\right)=\cos \left(\frac{\pi}{\operatorname{lcm}\left(6, N_{2}\right)}\right) .
$$

The different values of $\varphi$ in (31) yield the same CFA, up to a shift of the origin $\mathbf{c f a}[\mathbf{0}]$, a mirror symmetry or a permutation of the $R, G, B$ channels. So we assume $\varphi=\varphi_{0}$ in the following.

From (33), $\gamma_{C}$ is maximal if $N_{2}=3$ (which yields $n_{2}=1$ and $\omega_{0}=2 \pi / 3$ ). We ignore the second solution $N_{2}=6$ (with $\omega_{0}=\pi / 3$ ) because its modulation frequency is closer to $\mathbf{0}$ and all its other characteristics are the same as for $N_{2}=3$.
The last parameter is $\gamma_{L}$. From (30), we see that $\gamma_{C}$ and $\gamma_{L}$ are antagonist. For the minimal value $\gamma_{L}=\sqrt{3} / 2$, we have $\gamma_{C}=1 / 2$ and increasing $\gamma_{L}$ reduces $\gamma_{C}$ further. So, we fix $\gamma_{L}=\sqrt{3} / 2$, since a higher value would yield a much higher noise amplification in the chrominance channels than in the luminance of the demosaicked image.

\section{REFERENCES}

[1] L. Condat, "A new color filter array with optimal sensing properties," in Proc. of IEEE ICIP, Cairo, Egypt, Nov. 2009.

[2] K. Hirakawa and P. J. Wolfe, "Spatio-spectral color filter array design for optimal image recovery," IEEE Trans. Image Processing, vol. 17, no. 10 , pp. $1876-1890$, Oct. 2008.

[3] M. Kumar, E. Morales, J. Adams, and W. Hao, "New digital camera sensor architecture for low light imaging," in Proc. of IEEE ICIP, Cairo, Egypt, Nov. 2009.

[4] Y. M. Lu and M. Vetterli, "Optimal color filter array design: Quantitative conditions and an efficient search procedure," in Proc. of SPIE Conf. Digital Photography V, San Jose, CA, USA, Jan. 2009.

[5] L. Condat, "A new random color filter array with good spectral properties," in Proc. of IEEE ICIP, Cairo, Egypt, Nov. 2009.

[6] P. Hao, Y. Li, Z. Lin, and E. Dubois, "A geometric method for optimal design of color filter arrays," IEEE Trans. Image Processing, submitted.

[7] B. K. Gunturk, J. Glotzbach, Y. Altunbasak, R. W. Schaffer, and R. M. Mersereau, "Demosaicking: Color filter array interpolation," IEEE Signal Processing Mag., vol. 22, no. 1, pp. 44-54, Jan. 2005.

[8] K. Hirakawa and P. J. Wolfe, "Advancing the digital camera pipeline for mobile multimedia: Key challenges from a signal processing perspective," in Proc. of IEEE ICASSP, 5332-5335 2008.

[9] X. Li, B. K. Gunturk, and L. Zhang, "Image demosaicing: A systematic survey," in Proc. of IS\&T/SPIE VCIP, vol. 6822, San Jose, CA, USA, Jan. 2008.

[10] D. Menon, "Color image reconstruction for digital cameras," Ph.D. dissertation, Univ. Padova, Italy, 2008.

[11] D. Alleyson, S. Susstrunk, and J. Herault, "Linear demosaicing inspired by the human visual system," IEEE Trans. Image Processing, vol. 14, no. 4, pp. 439-449, Apr. 2005.

[12] T. Kijima, H. Nakamura, J. T. Compton, J. F. Hamilton, and T. E. DeWeese, "Image sensor with improved light sensitivity," U.S. Patent 0268533 , Nov., 2007

[13] M. Parmar and S. J. Reeves, "A perceptually based design methodology for color filter arrays," in Proc. of IEEE ICASSP, vol. 3, May 2004, pp. 473-476.

[14] W. Zhu, K. Parker, and M. A. Kriss, "Color filter arrays based on mutually exclusive blue noise patterns," Journal of Visual Communication and Image Representation, vol. 10, no. 3, pp. 245-267, Sept. 1999. 
[15] R. Lukac and K. N. Plataniotis, "Color filter arrays: Design and performance analysis," IEEE Trans. Consumer Electron., vol. 51, no. 4, pp. 1260-1267, Nov. 2005.

[16] Y. Li, P. Hao, and Z. Lin, "Color filter arrays: Representation and analysis," Dept of Computer Science, Queen Mary, University of London, Tech. Rep. RR-08-04, May 2008.

[17] — "Color filter arrays: A design methodology," Dept of Computer Science, Queen Mary, University of London, Tech. Rep. RR-08-03, May 2008.

[18] A. Davies and P. Fennessy, Digital imaging for photographers, 4th ed. Focal Press, 2001.

[19] A. Foi, M. Trimeche, V. Katkovnik, and K. Egiazarian, "Practical poissonian-gaussian noise modeling and fitting for single-image rawdata," IEEE Trans. Image Processing, vol. 17, no. 10, pp. 1737-1754, Oct. 2008.

[20] Y. Hel-Or, "The canonical correlations of color images and their use for demosaicing," HP Laboratories Israel, Tech. Rep. HPL-2003-164R1, Feb. 2004.

[21] J. Portilla, D. Otaduy, and C. Dorronsoro, "Low-complexity linear demosaicing using joint spatial-chromatic image statistics," in Proc. of IEEE ICIP, Sept. 2005.

[22] S. C. Pei and I. K. Tam, "Effective color interpolation in CCD color filter arrays using signal correlation," IEEE Trans. Circuits Syst. Video Technol., vol. 13, pp. 503-513, 2003.

[23] B. A. Wandell, Foundations of Vision. Sinauer Associates, Inc., 1995.

[24] E. Dubois, "Frequency-domain methods for demosaicking of Bayersampled color images," IEEE Signal Processing Lett., vol. 12, no. 12, pp. 847-850, Dec. 2005.

[25] K. Hirakawa and P. Wolfe, "Second-generation color filter array and demosaicking designs," in Proc. of SPIE VCIP, vol. 6822, 2008.

[26] E. Dubois, "Filter design for adaptive frequency-domain Bayer demosaicking," in Proc. of IEEE ICIP, Atlanta, USA, Oct. 2006, pp. 27052708 .

[27] B. C. de Lavarène, D. Alleysson, B. Durette, and J. Hérault, "Efficient demosaicing using recursive filtering," in Proc. of IEEE ICIP, San Antonio, USA, Sept. 2007.

[28] D. Alleysson, L. Meylan, and S. Süsstrunk, "HDR CFA image rendering," in Proc. of Eusipco, Sept. 2006.

[29] A. Foi, "Clipped noisy images: heteroskedastic modeling and practical denoising," Signal Processing, vol. 89, no. 12, pp. 2609-2629, Dec. 2009

[30] L. Condat, "A simple, fast and efficient approach to denoisaicking: Joint demosaicking and denoising," 2010, submitted to the conference IEEE ICIP. Preprint available online.

[31] L. Nai-Xiang, C. Lanlan, T. Yap-Peng, and V. Zagorodnov, "Adaptive filtering for color filter array demosaicking," IEEE Trans. Image Processing, vol. 16, no. 10, pp. 2515-2525, Oct. 2007.

[32] K. Dabov, A. Foi, V. Katkovnik, and K. Egiazarian, "Image denoising by sparse 3D transform-domain collaborative filtering," IEEE Trans. Image Processing, vol. 16, no. 8, pp. 2080-2095, Aug. 2007.

[33] A. Adams, N. Gelfand, J. Dolson, and M. Levoy, "Gaussian KD-trees for fast high-dimensional filtering," in Proc. of ACM SIGGRAPH, New Orleans, Louisiana, USA, 2009.

[34] A. Barbu, "Training an active random field for real-time image denoising," IEEE Trans. Image Processing, vol. 18, no. 11, pp. 2451-2462, Nov. 2009.

[35] M. Parmar and S. J. Reeves, "Selection of optimal spectral sensitivity functions for color filter arrays," in Proc. of IEEE ICIP, Oct. 2006, pp. 1005-1008. 